\title{
On Exact Series Solution for Strongly Coupled Mixed Parabolic Boundary Value Problems
}

\author{
Vicente Soler, ${ }^{1}$ Emilio Defez, ${ }^{2}$ and José Antonio Verdoy ${ }^{2}$ \\ ${ }^{1}$ Departamento de Matemática Aplicada, Universitat Politècnica de València, Camino de Vera s/n, 46022 Valencia, Spain \\ ${ }^{2}$ Instituto de Matemática Multidisciplinar, Universitat Politècnica de València, Camino de Vera s/n, 46022 Valencia, Spain \\ Correspondence should be addressed to Emilio Defez; edefez@imm.upv.es
}

Received 27 November 2013; Revised 6 February 2014; Accepted 9 February 2014; Published 3 April 2014

Academic Editor: Shengqiang Liu

Copyright (c) 2014 Vicente Soler et al. This is an open access article distributed under the Creative Commons Attribution License, which permits unrestricted use, distribution, and reproduction in any medium, provided the original work is properly cited.

This paper continues with the construction of the exact solution for parabolic coupled systems of the type $u_{t}=A u_{x x}, A_{1} u(0, t)+$ $B_{1} u_{x}(0, t)=0, A_{2} u(l, t)+B_{2} u_{x}(l, t)=0,0<x<1, t>0$, and $u(x, 0)=f(x)$, where $A_{1}, A_{2}, B_{1}$, and $B_{2}$ are arbitrary matrices for which the block matrix $\left(\begin{array}{ll}A_{1} & B_{1} \\ A_{2} & B_{2}\end{array}\right)$ is nonsingular, and $A$ is a positive stable matrix. Although this problem has been solved in the literature (Soler et al., 2013), in this work we are using completely new conditions.

\section{Introduction}

Coupled partial differential systems with coupled boundaryvalue conditions are frequent in different areas of science and technology, as in chemical physics [1-3], in scattering problems in quantum mechanics [4-6], thermoelastoplastic modelling [7], coupled diffusion problems [8-10], and so forth.

In [11], eigenfunctions of problems of the type

$$
\begin{aligned}
& u_{t}(x, t)=A u_{x x}(x, t), \quad 0<x<1, t>0, \\
& A_{1} u(0, t)+B_{1} u_{x}(0, t)=0 \in \mathbb{C}^{m}, \quad t>0, \\
& A_{2} u(1, t)+B_{2} u_{x}(1, t)=0 \in \mathbb{C}^{m}, \quad t>0,
\end{aligned}
$$

where the unknown $u=\left(u_{1}, u_{2}, \ldots, u_{m}\right)^{T}$ is a $m$-dimensional vector, are constructed under the following hypotheses.

(i) The matrix coefficient $A$ is a matrix which satisfies the following condition:

$$
\operatorname{Re}(z)>0 \text { for all eigenvalues } z \text { of } A \text {, }
$$

and thus $A$ is a positive stable matrix (where $\operatorname{Re}(z)$ denotes the real part of $z \in \mathbb{C}$ ). (ii) Matrices $A_{i}, B_{i}, i=1,2$, are $m \times m$ complex matrices, and we assume that the block matrix

$$
\left(\begin{array}{ll}
A_{1} & B_{1} \\
A_{2} & B_{2}
\end{array}\right) \text { is regular, }
$$

and also the matrix pencil

$$
A_{1}+\rho B_{1} \text { is regular. }
$$

Observe that condition (4) involves the existence of some $\rho_{0} \epsilon$ $\mathbb{C}$, matrix $A_{1}+\rho_{0} B_{1}$ being invertible [12].

In order to construct the eigenfunctions in [11], the following matrices $\widetilde{A}_{1}$ and $\widetilde{B}_{1}$ were defined by

$$
\widetilde{A}_{1}=\left(A_{1}+\rho_{0} B_{1}\right)^{-1} A_{1}, \quad \widetilde{B}_{1}=\left(A_{1}+\rho_{0} B_{1}\right)^{-1} B_{1},
$$

thus satisfying the condition

$$
\widetilde{A}_{1}+\rho_{0} \widetilde{B}_{1}=I,
$$

where matrix $I$ denotes, as usual, the identity matrix. Under hypothesis (3), matrix $B_{2}-\left(A_{2}+\rho_{0} B_{2}\right) \widetilde{B}_{1}$ is regular; see [11, page 431], and $\widetilde{A}_{2}$ and $\widetilde{B}_{2}$ are the matrices defined by

$$
\begin{gathered}
\widetilde{A}_{2}=\left[B_{2}-\left(A_{2}+\rho_{0} B_{2}\right) \widetilde{B}_{1}\right]^{-1} A_{2}, \\
\widetilde{B}_{2}=\left[B_{2}-\left(A_{2}+\rho_{0} B_{2}\right) \widetilde{B}_{1}\right]^{-1} B_{2},
\end{gathered}
$$


which satisfy the following conditions:

$$
\widetilde{B}_{2}-\left(\widetilde{A}_{2}+\rho_{0} \widetilde{B}_{2}\right) \widetilde{B}_{1}=I, \quad \widetilde{B}_{2} \widetilde{A}_{1}-\widetilde{A}_{2} \widetilde{B}_{1}=I .
$$

In [11] authors also assumed the following essential hypothesis:

exist $b_{1} \in \sigma\left(\widetilde{B}_{1}\right)-\{0\}, \quad b_{2} \in \sigma\left(\widetilde{B}_{2}\right), \quad v \in \mathbb{C}^{m}-\{0\}$,

such that $\left(\widetilde{B}_{1}-b_{1} I\right) v=\left(\widetilde{B}_{2}-b_{2} I\right) v=0$,

where $\sigma(C)$ denotes the set of all the eigenvalues of a matrix $C$ in $\mathbb{C}^{m \times m}$. These eigenfunctions introduced in [11] were also used in [13] to construct a series solution of the initial-value problem:

$$
\begin{gathered}
u_{t}(x, t)=A u_{x x}(x, t), \quad 0<x<1, t>0, \\
A_{1} u(0, t)+B_{1} u_{x}(0, t)=0, \quad t>0, \\
A_{2} u(1, t)+B_{2} u_{x}(1, t)=0, \quad t>0, \\
u(x, 0)=f(x), \quad 0 \leq x \leq 1,
\end{gathered}
$$

where $f(x)=\left(f_{1}(x), f_{2}(x), \ldots, f_{m}(x)\right)^{T}$ is an $m$-dimensional vector, under the additional hypothesis:

$$
\operatorname{Ker}\left(\widetilde{B}_{1}-b_{1} I\right) \bigcap \operatorname{Ker}\left(\widetilde{B}_{2}-b_{2} I\right)
$$

is an invariant subspace with respect to matrix $A$,

where a subspace $E$ of $\mathbb{C}^{m}$ is invariant by the matrix $A \in$ $\mathbb{C}^{m \times m}$, if $A(E) \subset E$.

It is not difficult to show examples where this assumption (9) is held but (14) is not held. Let us consider the following example:

Example 1. We will consider the homogeneous parabolic problem with homogeneous conditions (10)-(13), where matrix $A \in \mathbb{C}^{4 \times 4}$ is chosen:

$$
A=\left(\begin{array}{cccc}
2 & 1 & 0 & -1 \\
1 & 2 & 0 & -2 \\
-1 & 0 & 2 & 1 \\
0 & 0 & 0 & 1
\end{array}\right)
$$

and the $4 \times 4$ matrices $A_{i}, B_{i}, i \in\{1,2\}$, are

$$
\begin{aligned}
A_{1}=\left(\begin{array}{llll}
0 & 0 & 0 & 0 \\
0 & 0 & 0 & 0 \\
0 & 0 & 1 & 0 \\
0 & 0 & 0 & 1
\end{array}\right), & A_{2}=\left(\begin{array}{llll}
0 & 1 & 0 & 0 \\
1 & 0 & 0 & 0 \\
0 & 0 & 0 & 1 \\
0 & 0 & 0 & 0
\end{array}\right) \\
B_{1}=\left(\begin{array}{llll}
1 & 0 & 0 & 0 \\
0 & 1 & 0 & 0 \\
0 & 0 & 0 & 0 \\
0 & 0 & 0 & 0
\end{array}\right), & B_{2}=\left(\begin{array}{llll}
1 & 0 & 0 & 0 \\
1 & 0 & 0 & 0 \\
0 & 0 & 1 & 0 \\
0 & 0 & 0 & 1
\end{array}\right) .
\end{aligned}
$$

Due to (5)-(7) we obtain

$$
\begin{aligned}
\widetilde{A_{1}} & =\left(A_{1}+\rho_{0} B_{1}\right)^{-1} A_{1}=A_{1}, \\
\widetilde{B_{1}} & =\left(A_{1}+\rho_{0} B_{1}\right)^{-1} B_{1}=B_{1}, \\
\widetilde{A_{2}} & =\left(B_{2}-\left(A_{2}+\rho_{0} B_{2}\right) \widetilde{B_{1}}\right)^{-1} A_{2} \\
& =\left(\begin{array}{cccc}
-1 & 0 & 0 & 0 \\
0 & -1 & 0 & 0 \\
0 & 0 & 0 & 1 \\
0 & 0 & 0 & 0
\end{array}\right), \\
\widetilde{B_{2}} & =\left(B_{2}-\left(A_{2}+\rho_{0} B_{2}\right) \widetilde{B_{1}}\right)^{-1} B_{2} \\
& =\left(\begin{array}{cccc}
-1 & 0 & 0 & 0 \\
-1 & 0 & 0 & 0 \\
0 & 0 & 1 & 0 \\
0 & 0 & 0 & 1
\end{array}\right) .
\end{aligned}
$$

It is easy to verify that $\sigma\left(\widetilde{B}_{1}\right)=\{0,1\}$ and $\sigma\left(\widetilde{B}_{2}\right)=$ $\{1,0,-1\}$. If we take $b_{1}=1$, one gets

$$
\operatorname{Ker}\left(\widetilde{B}_{1}-I\right)=\left\langle\left\{\left(\begin{array}{l}
1 \\
0 \\
0 \\
0
\end{array}\right),\left(\begin{array}{l}
0 \\
1 \\
0 \\
0
\end{array}\right)\right\}\right\rangle .
$$

Taking into account that $\sigma\left(\widetilde{B}_{2}\right)$, we will have three possible values for $b_{2}$.

(i) For $b_{2}=1$ there is

$$
\operatorname{Ker}\left(\widetilde{B}_{2}-I\right)=\left\langle\left\{\left(\begin{array}{l}
0 \\
0 \\
1 \\
0
\end{array}\right),\left(\begin{array}{l}
0 \\
0 \\
0 \\
1
\end{array}\right)\right\}\right\rangle,
$$

and then condition (9) is not fulfilled.

(ii) For $b_{2}=0$, one gets

$$
\operatorname{Ker}\left(\widetilde{B}_{2}\right)=\left\langle\left\{\left(\begin{array}{l}
0 \\
1 \\
0 \\
0
\end{array}\right)\right\}\right\rangle .
$$

Thus, condition (9) is satisfied with

$$
v=\left(\begin{array}{l}
0 \\
1 \\
0 \\
0
\end{array}\right) \in \operatorname{Ker}\left(\widetilde{B}_{1}-I\right) \bigcap \operatorname{Ker}\left(\widetilde{B}_{2}\right)=\left\{\left(\begin{array}{l}
0 \\
1 \\
0 \\
0
\end{array}\right)\right\} .
$$

Next, let us verify if subspace $\operatorname{Ker}\left(\widetilde{B}_{1}-I\right) \bigcap \operatorname{Ker}\left(\widetilde{B}_{2}\right)$ is an invariant subspace for $A$. Let $x \in \operatorname{Ker}\left(\widetilde{B}_{1}-I\right) \bigcap \operatorname{Ker}\left(\widetilde{B}_{2}\right)$; then $x$ takes the form $x=\left(\begin{array}{l}0 \\ \lambda \\ 0 \\ 0\end{array}\right), \lambda \in \mathbb{C}$. In this case we obtain

$$
A x=\left(\begin{array}{c}
\lambda \\
2 \lambda \\
0 \\
0
\end{array}\right) \notin \operatorname{Ker}\left(\widetilde{B}_{1}-I\right) \bigcap \operatorname{Ker}\left(\widetilde{B}_{2}\right) .
$$


(iii) For $b_{2}=-1$ see

$$
\operatorname{Ker}\left(\widetilde{B}_{2}+I\right)=\left\langle\left\{\left(\begin{array}{l}
1 \\
1 \\
0 \\
0
\end{array}\right)\right\}\right\rangle .
$$

Thus, condition (9) is fulfilled with $v=\left(\begin{array}{l}1 \\ 1 \\ 0 \\ 0\end{array}\right) \in \operatorname{Ker}\left(\widetilde{B}_{1}-\right.$ I) $\bigcap \operatorname{Ker}\left(\widetilde{B}_{2}+I\right)$.

Now, we verify if subspace $\operatorname{Ker}\left(\widetilde{B}_{1}-I\right) \bigcap \operatorname{Ker}\left(\widetilde{B}_{2}+I\right)$ is an invariant subspace for $A$. Let $x \in \operatorname{Ker}\left(\widetilde{B}_{1}-I\right) \bigcap \operatorname{Ker}\left(\widetilde{B}_{2}+I\right)$; then $x$ takes the form $x=\left(\begin{array}{l}\lambda \\ \lambda \\ 0 \\ 0\end{array}\right), \lambda \in \mathbb{C}$. In this case we have

$$
A x=\left(\begin{array}{c}
3 \lambda \\
3 \lambda \\
-\lambda \\
0
\end{array}\right) \notin \operatorname{Ker}\left(\widetilde{B}_{1}-I\right) \bigcap \operatorname{Ker}\left(\widetilde{B}_{2}+I\right) \text {. }
$$

Thus, condition (14) is not fulfilled.

Observe that, in this example, hypothesis (9) is satisfied but in (14) it is not satisfied. Thus, the method proposed in [13] cannot be used to solve this problem.

This paper deals with the construction of eigenfunctions of problem (10)-(12) by assuming hypotheses (2), (3), and (4) but not hypothesis (9). This set of eigenfunctions allows us to construct a series solution of the problem (10)-(13). We provide conditions for the function $f(x)$ and the matrix coefficients, in order to ensure the existence of a series solution of the problem.

The paper is organized as follows: in Section 2 a set of eigenfunctions will be constructed under a new condition, different from condition (9); in Section 3 a series solution for the problem is presented. In Section 4 we will introduce an algorithm and give an illustrative example.

Throughout this paper we will assume the results and nomenclature given in [11]. If $B$ is a matrix in $\mathbb{C}^{n \times m}$, we denote by $B^{\dagger}$ its Moore-Penrose pseudoinverse [12]. A collection of examples, properties, and applications of this concept may be found in [14], and $B^{\dagger}$ can be efficiently computed with the MATLAB and Mathematica computer algebra systems.

\section{The New Conditions}

In Section 2 of [11] the eigenfunctions of problem (10)(12) were constructed by using a matrix variable separation technique. We can repeat the calculations in this section to reach condition (44):

$$
\lambda \cot (\lambda) \in \sigma\left(-\widetilde{A}_{2} \widetilde{A}_{1}-\lambda^{2} \widetilde{B}_{2} \widetilde{B}_{1}\right) .
$$

Instead of (9), we will assume the following new condition:

$$
0 \in \sigma\left(\widetilde{B}_{1}\right), \quad a_{2} \in \sigma\left(\widetilde{A}_{2}\right),
$$

and we have $w \in \mathbb{C}^{m}-\{0\}$,

being $\widetilde{B}_{1} w=\left(\widetilde{A}_{2}-a_{2} I\right) w=0$.

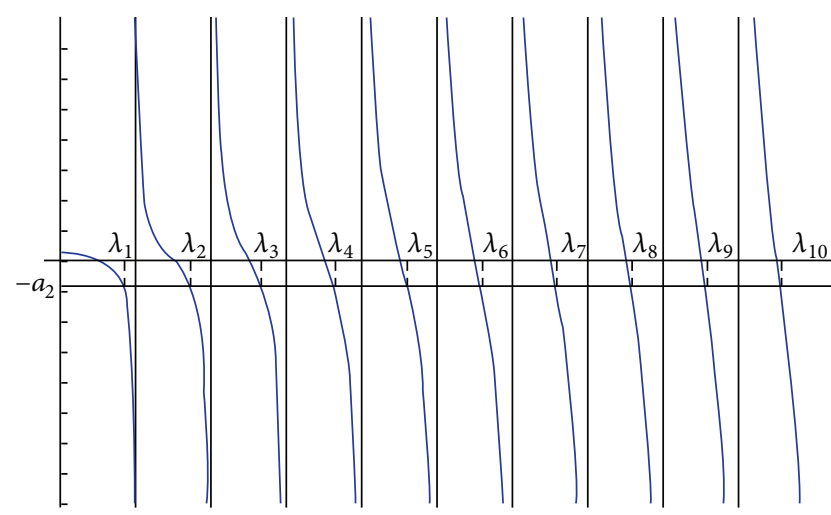

FIGURE 1: Graphical representation of $y=\lambda \cot (\lambda)$ and determination of the eigenvalues $\lambda_{n}$ for $-a_{2}<0$.

From relation (6) $\widetilde{A}_{1} w=w$ is obtained (because, obviously, $\left.\widetilde{B}_{1} w=0\right)$. Considering (8) $\widetilde{B}_{2} w=w$ is obtained. Thus $\left(-\widetilde{A}_{2} \widetilde{A}_{1}-\lambda^{2} \widetilde{B}_{2} \widetilde{B}_{1}\right) w=-a_{2} w$; that is, $-a_{2}$ is the eigenvalue which will be equal to $\lambda \cot (\lambda)$ in (26):

$$
\lambda \cot (\lambda)=-a_{2} .
$$

Let us assume that $a_{2}$ given in (28) satisfies

$$
a_{2} \in \mathbb{R} .
$$

We will observe that, under hypothesis (29), we have guaranteed the existence of the solutions for the equation

$$
\lambda \cot (\lambda)=-a_{2} \text {. }
$$

Equation (30) has a unique solution $\lambda_{k}$ in each interval $(k \pi,(k+1) \pi)$ for $k \geq 1$, as seen in Figure 1. Also, the following lemma is easily demonstrated.

Lemma 2. Under hypothesis (29), the roots $\lambda_{k}$ of (30) fulfil $\lim _{n \rightarrow \infty} \lambda_{n}=+\infty$. Also,

$$
\begin{gathered}
\lim _{n \rightarrow \infty}\left|\sin \left(\lambda_{n}\right)\right|=1, \quad \lim _{n \rightarrow \infty} \cos \left(\lambda_{n}\right)=0, \\
\lim _{n \rightarrow \infty}\left(\lambda_{n+1}-\lambda_{n}\right)=\pi .
\end{gathered}
$$

Proof. Function $f(\lambda)=\lambda \cot (\lambda)$ has got vertical asymptotes at the points $\lambda=k \pi, k \in \mathbb{N}$ and $f(\lambda)$ has got zeros at the points $\lambda=\pi / 2+k \pi, k \in \mathbb{N}$. Therefore, as we have stated, the real coefficient function $-a_{2}$ intersects the graph of the function $f(\lambda)$ in each interval $(k \pi,(k+1) \pi)$, where $\lambda_{k} \in(k \pi,(k+1) \pi)$ is the point of intersection. Therefore, the sequence $\left\{\lambda_{k}\right\}_{k \geq 1}$ is monotone increasing with $\lim _{k \rightarrow \infty} \lambda_{k}=\infty$. First, we have to consider two possibilities.

(i) If $-a_{2}>0$, as can be seen in Figure 1, for $k$ being large enough, $\lambda_{k} \in(k \pi, \pi / 2+k \pi)$.

(ii) If $-a_{2}<0$, as can be seen in Figure 1, for $k$ being large enough, $\lambda_{k} \in(\pi / 2+k \pi,(k+1) \pi)$.

Therefore, observe that $\pi / 2<\lambda_{k+1}-\lambda_{k}<3 \pi / 2$ for $k$ being large enough. For $\lambda_{k}$, substituted in (30), we get

$$
\lambda_{k} \cot \left(\lambda_{k}\right)=-a_{2} \text {, }
$$


by dividing by $\lambda_{k}$. By taking limits where $k \rightarrow \infty$, $\lim _{k \rightarrow \infty} \cot \left(\lambda_{k}\right)=0$ is obtained, and in this way the sequence $\left\{\sin \left(\lambda_{k}\right)\right\}_{k \geq 1}$ is bounded, $\lim _{k \rightarrow \infty} \cos \left(\lambda_{k}\right)=0$, and $\lim _{k \rightarrow \infty}\left|\sin \left(\lambda_{k}\right)\right|=1$. Moreover,

$$
\cot \left(\lambda_{k+1}-\lambda_{k}\right)=\frac{\cot \left(\lambda_{k+1}\right) \cot \left(\lambda_{k}\right)+1}{\cot \left(\lambda_{k}\right)-\cot \left(\lambda_{k+1}\right)},
$$

so, considering limits where $k \rightarrow \infty$, one gets $\lim _{k \rightarrow \infty} \cot \left(\lambda_{k+1}-\lambda_{k}\right)=\infty$, and the sequence $\left\{\cos \left(\lambda_{k+1}-\right.\right.$ $\left.\left.\lambda_{k}\right)\right\}_{k \geq 1}$ is also bounded. Moreover $\lim _{k \rightarrow \infty} \sin \left(\lambda_{k+1}-\lambda_{k}\right)=$ 0 , and with $\pi / 2<\lambda_{k+1}-\lambda_{k}<3 \pi / 2$, one gets that $\lim _{k \rightarrow \infty}\left(\lambda_{k+1}-\lambda_{k}\right)=\pi$.

Finally, if $a_{2}=0,(30)$ is reduced to $\lambda \cot (\lambda)=0$, whose roots are $\lambda_{k}=\pi / 2+k \pi, k \in \mathbb{N}$, and trivially $\lambda_{k+1}-\lambda_{k}=\pi$, then $\lim _{k \rightarrow \infty}\left(\lambda_{k+1}-\lambda_{k}\right)=\pi$.

Observe that under hypothesis $-a_{2}<1$ there is a root $\lambda_{0} \in(0, \pi)$, and we can define the set of eigenvalues of the problem (10)-(12) as

$$
\mathscr{F}=\left\{\lambda_{k} \in(k \pi,(k+1) \pi) ; \lambda_{k} \cot \left(\lambda_{k}\right)=-a_{2}, k \geq 1\right\} \cup \mathscr{F}_{0},
$$

where

$$
\mathscr{F}_{0}= \begin{cases}\emptyset, & \text { if }-a_{2}>1 \\ \{0\}, & \text { if }-a_{2}=1 \\ \lambda_{0} \in(0, \pi), & \text { if }-a_{2}<1 .\end{cases}
$$

Thus, by [11, page 433] a set of solutions of problem (10)-(12) is given by

$$
\begin{array}{r}
u\left(x, t, \lambda_{k}\right)=e^{-\lambda_{k} A t}\left\{\sin \left(\lambda_{k} x\right) \widetilde{A}_{1}-\lambda_{k} \cos \left(\lambda_{k} x\right) \widetilde{B}_{1}\right\} C\left(\lambda_{k}\right), \\
\lambda_{k} \in \mathscr{F},
\end{array}
$$

where $C\left(\lambda_{k}\right)$ satisfies

$$
\begin{gathered}
\left(\widetilde{B}_{1} A^{j}-A^{j} \widetilde{B}_{1}\right) C\left(\lambda_{k}\right)=0, \quad 0<j<p, \\
\left(-a_{2} I+\widetilde{A}_{2} \widetilde{A}_{1}+\lambda^{2} \widetilde{B}_{2} \widetilde{B}_{1}\right) A^{j} C\left(\lambda_{k}\right)=0, \quad 0 \leq j<p,
\end{gathered}
$$

and where $p$ is the degree of minimal polynomial of $A$. Formulas (37) are equivalent to the matrix equation:

$$
G_{\lambda_{k}}\left(\rho_{0}\right) C\left(\lambda_{k}\right)=0,
$$

where

$$
G_{\lambda_{k}}\left(\rho_{0}\right)=\left(\begin{array}{c}
\widetilde{B}_{1} A-A \widetilde{B}_{1} \\
\vdots \\
\widetilde{B}_{1} A^{p-1}-A^{p-1} \widetilde{B}_{1} \\
\widetilde{A}_{2} \widetilde{A}_{1}+\lambda_{k}^{2} \widetilde{B}_{2} \widetilde{B}_{1}-a_{2} I \\
\left(\widetilde{A}_{2} \widetilde{A}_{1}+\lambda_{k}^{2} \widetilde{B}_{2} \widetilde{B}_{1}-a_{2} I\right) A \\
\vdots \\
\left(\widetilde{A}_{2} \widetilde{A}_{1}+\lambda_{k}^{2} \widetilde{B}_{2} \widetilde{B}_{1}-a_{2} I\right) A^{p-1}
\end{array}\right) .
$$

In order to ensure that $C\left(\lambda_{k}\right) \neq 0$ fulfils (37) we have

$$
\operatorname{rank} G_{\lambda_{k}}\left(\rho_{0}\right)<m \text {, }
$$

and under condition (40), the solution of (37) is given by

$$
C\left(\lambda_{k}\right)=\left(I-G_{\lambda_{k}}\left(\rho_{0}\right)^{\dagger} G_{\lambda_{k}}\left(\rho_{0}\right)\right) S, \quad S \in \mathbb{C}^{m} .
$$

The eigenfunctions associated to the problem (10)-(12) are then given by

$$
\begin{array}{r}
u\left(x, t, \lambda_{k}\right)=e^{-\lambda_{k} A t}\left\{\sin \left(\lambda_{k} x\right) \widetilde{A}_{1}-\lambda_{k} \cos \left(\lambda_{k} x\right) \widetilde{B}_{1}\right\} C\left(\lambda_{k}\right), \\
\lambda_{k} \in \mathscr{F} .
\end{array}
$$

Working in a similar form to that in [11, page 433], we can show that also $\lambda=0$ is an eigenvalue of problem (10), if

$$
1 \in \sigma\left(-\widetilde{A}_{2} \widetilde{A}_{1}\right) \text {. }
$$

Under hypothesis (43), if we denote

$$
C(0)=\left(I-G_{0}\left(\rho_{0}\right)^{\dagger} G_{0}\left(\rho_{0}\right)\right) S, \quad S \in \mathbb{C}^{m},
$$

one gets that function

$$
u(x, 0)=\left(x \widetilde{A}_{1}-\widetilde{B}_{1}\right) C(0)
$$

is an eigenfunction of problem (10) associated to eigenvalue $\lambda=0$.

As a conclusion, the following theorem has been demonstrated.

Theorem 3. Consider problem (10)-(12) which fulfils conditions (2) and (3). Let $p$ be the degree of the minimal polynomial of matrix $A$ and let $\rho_{0}$ be a real number satisfying (4). Let $\widetilde{A}_{1}$, $\widetilde{B}_{1}$ be the matrices defined by (5) and $\widetilde{A}_{2}, \widetilde{B}_{2}$ by (7), respectively.

(1) Let us assume conditions (27) and (29). Then (28) admits a set of real positive solutions denoted by $\mathscr{F}$ and defined by (34). Let $G_{\lambda_{k}}\left(\rho_{0}\right)$ be the matrix defined by (39) where $\lambda_{k} \in \mathscr{F}$ and suppose that condition (40) is fulfilled. Then, problem (10)-(12) admits eigenfunctions $u\left(x, t, \lambda_{k}\right)$ associated to positive eigenvalues $\lambda_{k} \in \mathscr{F}$ and defined by (42), where $C\left(\lambda_{k}\right)$ is given by (41) where $S$ is a vector in $\mathbb{C}^{m}$.

(2) $\lambda=0$ is an eigenvalue of problem (10)-(12) if condition (43) is fulfilled. Under hypothesis (43), if $G_{0}\left(\rho_{0}\right)=$ $\widetilde{A_{2}} \widetilde{A}_{1}+I$, then expression (45) provides eigenfunction of problem (10)-(12) associated to eigenvalue $\lambda=0$.

\section{A Series Solution}

By assuming superposition principle, a possible series solution of problem (10)-(13) is given by

$$
u(x, t)= \begin{cases}u(x, 0)+\sum_{\lambda_{n} \in \mathscr{F}} u\left(x, t, \lambda_{k}\right), & 0 \in \mathscr{F}, \\ \sum_{\lambda_{n} \in \mathscr{F}} u\left(x, t, \lambda_{k}\right), & 0 \notin \mathscr{F},\end{cases}
$$


where $u\left(x, t, \lambda_{k}\right)$ and $u(x, 0)$ are defined by (42) and (45), respectively, for suitable vectors $C\left(\lambda_{n}\right)$ and $C(0)$.

Assuming that series (46) and the corresponding derivatives $u_{x}(x, t), u_{x x}(x, t)$, and $u_{t}(x, t)$ are convergent (we will demonstrate this later), (46) will be a solution of (10)-(12). Now, we need to determine vectors $C(\lambda)$ and $C(0)$ so that $(46)$ satisfies (13).

Note that, by taking $w$ to fulfil (27), from (8) we have

$$
\widetilde{A}_{2} w=a_{2} w, \quad \widetilde{A}_{1} w=w .
$$

Under condition (47), we will consider the scalar SturmLiouville problem:

$$
\begin{gathered}
X^{\prime \prime}(x)+\lambda^{2} X(x)=0, \\
X(0)=0, \\
a_{2} X(1)+X^{\prime}(1)=0,
\end{gathered}
$$

which provides a family of eigenvalues $\mathscr{F}$ given in (34). Then, the associated eigenfunctions are

$$
\begin{gathered}
X_{\lambda_{n}}(x)=\sin \left(\lambda_{n} x\right), \quad \lambda_{n}>0, \\
X_{0}(x)=x, \quad \text { if } \lambda_{0}=0 .
\end{gathered}
$$

According to the theorem of convergence of SturmLiouville for functional series [15, chapter 11], with the initial condition $f(x)=\left(f_{1}(x), \ldots, f_{m}(x)\right)^{t}$ given in (13) which fulfils the following properties:

$$
\begin{gathered}
f \in \mathscr{C}^{2}([0,1]), \\
f(0)=0, \\
a_{2} f(1)+f^{\prime}(1)=0,
\end{gathered}
$$

each component $f_{i}$ of $f$, for $1 \leq i \leq m$, has got a series expansion which converges absolutely and uniformly to the interval $[0,1]$; namely,

$$
f_{i}(x)=\alpha x e_{0 i}+\sum_{\lambda_{n} \in \mathscr{F}} \sin \left(\lambda_{n} x\right) e_{\lambda_{n} i}
$$

where

$$
\begin{gathered}
\alpha= \begin{cases}1, & \text { si } \lambda_{0}=0, \\
0, & \text { si } \lambda_{0} \neq 0,\end{cases} \\
e_{0 i}=\frac{\int_{0}^{1} x f_{i}(x) d x}{\int_{0}^{1} x^{2} d x} \text { if } \lambda_{0}=0, \\
e_{\lambda_{n} i}=\frac{\int_{0}^{1} \sin \left(\lambda_{n} x\right) f_{i}(x) d x}{\int_{0}^{1}\left(\sin \left(\lambda_{n} x\right)\right)^{2} d x} \text { if } \lambda_{n}>0 .
\end{gathered}
$$

Thus,

$$
f(x)=\alpha x E(0)+\sum_{\lambda_{n} \in \mathscr{F}} \sin \left(\lambda_{n} x\right) E\left(\lambda_{n}\right),
$$

where $E(0)=\left(\begin{array}{c}e_{01} \\ \vdots \\ e_{0 m}\end{array}\right)$ and $E\left(\lambda_{n}\right)=\left(\begin{array}{c}e_{\lambda_{n} 1} \\ \vdots \\ e_{\lambda_{n} m}\end{array}\right)$. On the other hand, from (46) and taking into account (42) and (45), we obtain

$$
\begin{aligned}
u(x, 0)= & \alpha\left(x \widetilde{A}_{1}-\widetilde{B}_{1}\right) C(0) \\
& +\sum_{\lambda_{n} \in \mathscr{F}}\left(\sin \left(\lambda_{n} x\right) \widetilde{A}_{1}-\lambda_{n} \cos \left(\lambda_{n} x\right) \widetilde{B}_{1}\right) C\left(\lambda_{n}\right) .
\end{aligned}
$$

We can equate the two expressions (53) and (54) if $C(0)$ and $C\left(\lambda_{n}\right)$, apart from conditions (41) and (44), satisfy $\{C(0), C(\lambda)\} \subset \operatorname{Ker}\left(\widetilde{B}_{1}\right)$. Then, we have

$$
\begin{gathered}
C\left(\lambda_{n}\right)=E\left(\lambda_{n}\right)=\frac{\int_{0}^{1} \sin \left(\lambda_{n} x\right) f(x) d x}{\int_{0}^{1}\left(\sin \left(\lambda_{n} x\right)\right)^{2} d x}, \quad \text { if } \lambda_{n}>0, \\
C(0)=E(0)=\frac{\int_{0}^{1} x f(x) d x}{\int_{0}^{1} x^{2} d x} \quad \text { if } \lambda_{0}=0 .
\end{gathered}
$$

Note $C(0)$ and $C(\lambda) \in \operatorname{Ker}\left(\widetilde{B}_{1}\right)$, if

$$
f(x) \in \operatorname{Ker}\left(\widetilde{B}_{1}\right) .
$$

Then $u(x, t)$ is defined by

$$
u(x, t)=\alpha x C(0)+\sum_{\lambda_{n} \in \mathscr{F}} e^{-\lambda_{n}^{2} A t} \sin \left(\lambda_{n} x\right) C\left(\lambda_{n}\right),
$$

where $\alpha$ and $C\left(\lambda_{n}\right)$ are defined by (52) and (55), respectively, and $u(x, y)$ fulfils the initial condition (13). Note that conditions (37)-(40) are held if

$$
A^{j} f(x) \in \operatorname{Ker}\left(\widetilde{B}_{1}\right) \cap \operatorname{Ker}\left(\widetilde{A}_{2}-a_{2} I\right), \quad 0 \leq j<p-1 .
$$

Condition (58) is equivalent to

$$
\begin{aligned}
& f(x) \in \operatorname{Ker}\left(\widetilde{B}_{1}\right) \cap \operatorname{Ker}\left(\widetilde{A}_{2}-a_{2} I\right), \quad 0 \leq x \leq 1, \\
& \operatorname{Ker}\left(\widetilde{B}_{1}\right) \cap \operatorname{Ker}\left(\widetilde{A}_{2}-a_{2} I\right)
\end{aligned}
$$

is an invariant subspace with respect to matrix $A$.

The study of the convergence of the series solution (57) with $\alpha$ defined by (52) and $C\left(\lambda_{n}\right)$ by (55), by using Lemma 2 , can be reduced to that made in [13] for the case $b_{1}=$ 0 . Similarly, independence of the series solution (57) with respect to the chosen $\rho_{0} \in \mathbb{R}$ can be demonstrated with the same technique as given in [16].

We can summarize the results in the following theorem.

Theorem 4. Consider the homogeneous problem with homogeneous conditions (10)-(13) under hypotheses given in Theorem 3. Assume that function $f$ of (13) fulfils conditions (50) and (59). Then, the series defined in (46) is a solution of problem (10)-(13). 
Input data: $A, A_{1}, A_{2}, B_{1}, B_{2} \in \mathbb{C}^{m \times m}, f(x) \in \mathbb{C}^{m}$.

Result: $u(x, t)$.

(1) Verify that matrix $A$ satisfies (2).

(2) Verify that matrices $A_{i}, B_{i} \in \mathbb{C}^{m \times m}, i \in\{1,2\}$ are singular, and check that the block matrix $\left(\begin{array}{ll}A_{1} & B_{1} \\ A_{2} & B_{2}\end{array}\right)$ is regular.

(3) Determine a number $\rho_{0} \in \mathbb{R}$ so that the matrix pencil $A_{1}+\rho_{0} B_{1}$ is regular.

(4) Determine matrices $\widetilde{A_{1}}$ and $\widetilde{B_{1}}$ defined in (5).

(5) Determine matrices $\widetilde{A_{2}}$ and $\widetilde{B_{2}}$ defined in (7).

(6) Consider the following cases:

(i) Case 1: Condition (27) is fulfilled, that is, matrices $\widetilde{B}_{1}$ and $\widetilde{A}_{2}$ have a common eigenvector $w \neq 0$ associated with eigenvalues $0 \in \sigma\left(\widetilde{B}_{1}\right)$ and $a_{2} \in \sigma\left(\widetilde{A}_{2}\right)$. In this case we will continue with step (7).

(ii) Case 2: Condition (27) is not fulfilled. In this case the algorithm is not completed because it is not possible to find the solution of (10)-(13) for the given data.

(7) Take $0 \in \sigma\left(\widetilde{B}_{1}\right)$ and determine $a_{2} \in \sigma\left(\widetilde{A}_{2}\right) \cap \mathbb{R}$, and vector $w \neq 0$ when $w \in \operatorname{Ker}\left(\widetilde{B}_{1}\right) \cap \operatorname{Ker}\left(\widetilde{A_{2}}\right)$ in a way that:

(i) Conditions (59) fulfilled, that is:

(1.1) $\operatorname{Ker}\left(\widetilde{B}_{1}\right) \bigcap \operatorname{Ker}\left(\widetilde{A}_{2}-a_{2} I\right)$ is an invariant subspace respect to matrix $A$.

(1.2) $f(x) \in \operatorname{Ker}\left(\widetilde{B}_{1}\right) \cap \operatorname{Ker}\left(\widetilde{A}_{2}-a_{2} I\right), \forall x \in[0,1]$.

(ii) The vectorial function $f(x)$ fulfils (50), that is:

(1.3) $f \in \mathscr{C}^{2}([0,1])$.

(1.4) $f(0)=0$.

(1.5) $a_{2} f(1)+f^{\prime}(1)=0$.

If these conditions are not satisfied, go back over step (6) of Algorithm (1) and discard the value taken for $a_{2}$.

(8) Determine the positive solutions of (30) and determine $\mathscr{F}$ defined in (34).

(9) Determine degree $p$ of minimal polynomial of matrix $A$.

(10) Build block matrix $G_{\lambda_{k}}\left(\rho_{0}\right)$ defined in (39).

(11) Determine $\lambda_{k} \in \mathscr{F}$ so that $\operatorname{rank} G_{\lambda_{k}}\left(\rho_{0}\right)<m$.

(12) Include the eigenvalue $\lambda=0$ if $1 \in \sigma\left(-\widetilde{A}_{2} \widetilde{A}_{1}\right)$.

(13) Determine $\alpha$ given in (52).

(14) Determine vectors $C\left(\lambda_{n}\right)$ defined in (55).

(15) Determine the series solution $u(x, t)$ of problem (10)-(13) defined in (57).

Algorithm 1: Solution of the homogeneous problem with homogeneous conditions (10)-(13).

\section{Algorithm and Example}

We can summarize the process to calculate the solution of the homogeneous problem with homogeneous conditions (10)(13) in Algorithm 1.

Example 5. We will consider the homogeneous parabolic problem with homogeneous conditions (10)-(13), given in Example 1, that is, where the matrix $A \in \mathbb{C}^{4 \times 4}$ is given in (15) and matrices $A_{i}, B_{i}, i \in\{1,2\}$ are given in (16). We consider the vector-valued function $f(x)$ to be defined as

$$
f(x)=\left(\begin{array}{c}
0 \\
0 \\
x^{2}-2 x \\
0
\end{array}\right)
$$

Observe that, as demonstrated in Example 1, hypothesis (9) is fulfilled but (14) is not fulfilled. Thus, the method proposed in [13] cannot be used to solve this problem.
Algorithm 1 (step by step). Consider

(1) Matrix $A$ satisfies the condition (2), because $\sigma(A)=$ $\{1,2,3\}$; that is, $A$ is positive stable.

(2) Each of the matrices $A_{i}, B_{i}, i \in\{1,2\}$ is singular, and the block matrix,

$$
\left(\begin{array}{ll}
A_{1} & B_{1} \\
A_{2} & B_{2}
\end{array}\right)=\left(\begin{array}{llll|llll}
0 & 0 & 0 & 0 & 1 & 0 & 0 & 0 \\
0 & 0 & 0 & 0 & 0 & 1 & 0 & 0 \\
0 & 0 & 1 & 0 & 0 & 0 & 0 & 0 \\
0 & 0 & 0 & 1 & 0 & 0 & 0 & 0 \\
\hline 0 & 1 & 0 & 0 & 1 & 0 & 0 & 0 \\
1 & 0 & 0 & 0 & 1 & 0 & 0 & 0 \\
0 & 0 & 0 & 1 & 0 & 0 & 1 & 0 \\
0 & 0 & 0 & 0 & 0 & 0 & 0 & 1
\end{array}\right),
$$

is regular.

(3) Note that although $A_{1}$ is singular, if we take $\rho_{0}=1 \epsilon$ $\mathbb{R}$, the matrix pencil,

$$
A_{1}+\rho_{0} B_{1}=I_{4 \times 4},
$$

is regular. Therefore, $\rho_{0}=1$. 
(4) In (17) we have

$$
\begin{gathered}
\widetilde{A_{1}}=\left(A_{1}+\rho_{0} B_{1}\right)^{-1} A_{1}=A_{1}, \\
\widetilde{B_{1}}=\left(A_{1}+\rho_{0} B_{1}\right)^{-1} B_{1}=B_{1} .
\end{gathered}
$$

(5) In (18) we have

$$
\begin{aligned}
\widetilde{A_{2}} & =\left(B_{2}-\left(A_{2}+\rho_{0} B_{2}\right) \widetilde{B_{1}}\right)^{-1} A_{2} \\
& =\left(\begin{array}{cccc}
-1 & 0 & 0 & 0 \\
0 & -1 & 0 & 0 \\
0 & 0 & 0 & 1 \\
0 & 0 & 0 & 0
\end{array}\right), \\
\widetilde{B_{2}} & =\left(B_{2}-\left(A_{2}+\rho_{0} B_{2}\right) \widetilde{B_{1}}\right)^{-1} B_{2} \\
& =\left(\begin{array}{cccc}
-1 & 0 & 0 & 0 \\
-1 & 0 & 0 & 0 \\
0 & 0 & 1 & 0 \\
0 & 0 & 0 & 1
\end{array}\right) .
\end{aligned}
$$

(6) Also $\sigma\left(\widetilde{B_{1}}\right)=\{0,1\}$ and $\sigma\left(\widetilde{A_{2}}\right)=\{0,-1\}$. Note that in this case the condition (27) is fulfilled because with $0 \in \sigma\left(\widetilde{B_{1}}\right)$ and $a_{2}=0 \in \sigma\left(\widetilde{A_{2}}\right)$ there is a common eigenvector $w \in \mathbb{C}^{4}, w=\left(\begin{array}{l}0 \\ 0 \\ 1 \\ 0\end{array}\right)$, and thus $\operatorname{Ker}\left(\widetilde{B}_{1}\right) \bigcap \operatorname{Ker}\left(\widetilde{A}_{2}\right) \neq\left(\begin{array}{l}0 \\ 0 \\ 0 \\ 0\end{array}\right)$. Therefore, we are in case 1 of Algorithm 1.

(7) We take the values $b_{1}=0, a_{2}=0$, and we will check the conditions given in step 7 of the algorithm:

$$
\operatorname{Ker}\left(\widetilde{B}_{1}\right) \bigcap \operatorname{Ker}\left(\widetilde{A}_{2}\right)=\left\langle\left(\begin{array}{l}
0 \\
0 \\
1 \\
0
\end{array}\right)\right\rangle .
$$

Let $x \in \operatorname{Ker}\left(\widetilde{B}_{1}\right) \bigcap \operatorname{Ker}\left(\widetilde{A}_{2}\right)$; then $x=\left(\begin{array}{l}0 \\ 0 \\ \lambda \\ 0\end{array}\right), \lambda \epsilon$

$\mathbb{C}$. In this case we have

$$
A x=\left(\begin{array}{c}
0 \\
0 \\
2 \lambda \\
0
\end{array}\right) \in \operatorname{Ker}\left(\widetilde{B}_{1}\right) \bigcap \operatorname{Ker}\left(\widetilde{A}_{2}\right) \text {, }
$$

and then the subspace $\operatorname{Ker}\left(\widetilde{B}_{1}\right) \bigcap \operatorname{Ker}\left(\widetilde{A}_{2}\right)$ is invariant by matrix $A$.

It is trivial to verify the following:

$f(x) \in \operatorname{Ker}\left(\widetilde{B}_{1}\right) \bigcap \operatorname{Ker}\left(\widetilde{A_{2}}\right), \quad \forall x \in[0,1]$.

(1.3) $f(x) \in \mathscr{C}^{2}([0,1])$,

$$
\begin{aligned}
& \text { (1.4) } f(0)=\left(\begin{array}{l}
0 \\
0 \\
0 \\
0
\end{array}\right), \\
& \text { (1.5) } a_{2} f(1)+f^{\prime}(1)=\left(\begin{array}{l}
0 \\
0 \\
0 \\
0
\end{array}\right) \text {, }
\end{aligned}
$$

(8) Equation (30) is as follows:

$$
\lambda \cot (\lambda)=0 .
$$

We can solve (68) exactly, $\lambda_{k}=\pi / 2+k \pi$, with an additional solution $\left.\lambda_{0} \in\right] 0, \pi[$, because

$$
-a_{2}=0<1 \text {, }
$$

and then $\lambda_{0}=\pi / 2$. Thus, we have a numerable family of solutions for (68) which we denote $\mathscr{F}$, given by

$$
\begin{array}{r}
\mathscr{F}=\left\{\lambda_{k}=\frac{\pi}{2}+k \pi ; \lambda_{k} \in(k \pi,(k+1) \pi), k \geq 1\right\} \cup \mathscr{F}_{0}, \\
\mathscr{F}_{0}=\left\{\lambda_{0}=\frac{\pi}{2}\right\} .
\end{array}
$$

(9) The minimal polynomial of matrix $A$ is given by $p(x)=(x-1)^{2}(x-3)(x-2)$, and then $p=4$.

(10) If $\lambda_{k}$ is a positive solution of (68), the matrix $G_{\lambda_{k}}\left(\rho_{0}\right)$ given by (39) takes the form

$$
G_{\lambda_{k}}\left(\rho_{0}\right)=\left(\begin{array}{cccc}
0 & 0 & 0 & -1 \\
0 & 0 & 0 & -2 \\
1 & 0 & 0 & 0 \\
0 & 0 & 0 & 0 \\
\hline 0 & 0 & 0 & -5 \\
0 & 0 & 0 & -7 \\
4 & 1 & 0 & 0 \\
0 & 0 & 0 & 0 \\
\hline 0 & 0 & 0 & -18 \\
0 & 0 & 0 & -21 \\
13 & 6 & 0 & 0 \\
0 & 0 & 0 & 0 \\
\hline-\lambda_{k}^{2} & 0 & 0 & 0 \\
-\lambda_{k}^{2} & 0 & 0 & 0 \\
0 & 0 & 0 & 1 \\
0 & 0 & 0 & 0 \\
\hline-2 \lambda_{k}^{2} & -\lambda_{k}^{2} & 0 & \lambda_{k}^{2} \\
-2 \lambda_{k}^{2} & -\lambda_{k}^{2} & 0 & \lambda_{k}^{2} \\
0 & 0 & 0 & 1 \\
0 & 0 & 0 & 0 \\
\hline-5 \lambda_{k}^{2} & -4 \lambda_{k}^{2} & 0 & 5 \lambda_{k}^{2} \\
-5 \lambda_{k}^{2} & -4 \lambda_{k}^{2} & 0 & 5 \lambda_{k}^{2} \\
0 & 0 & 0 & 1 \\
0 & 0 & 0 & 0 \\
\hline-14 \lambda_{k}^{2} & -13 \lambda_{k}^{2} & 0 & 18 \lambda_{k}^{2} \\
-14 \lambda_{k}^{2} & -13 \lambda_{k}^{2} & 0 & 18 \lambda_{k}^{2} \\
0 & 0 & 0 & 1 \\
0 & 0 & 0 & 0
\end{array}\right) .
$$

(11) Since the third column $G_{\lambda_{k}}\left(\rho_{0}\right)$ is zero, rank $G_{\lambda_{k}}$ $\left(\rho_{0}\right)<4$. Thus, each of the positive solutions given in (70) is an eigenvalue. 
(12) It is trivial to verify that $1 \notin \sigma\left(-\widetilde{A}_{2} \widetilde{A}_{1}\right)$, because

$$
-\widetilde{A}_{2} \widetilde{A}_{1}=\left(\begin{array}{cccc}
0 & 0 & 0 & 0 \\
0 & 0 & 0 & 0 \\
0 & 0 & 0 & -1 \\
0 & 0 & 0 & 0
\end{array}\right), \quad \sigma\left(-\widetilde{A}_{2} \widetilde{A}_{1}\right)=\{0\}
$$

and thus we will not include 0 as an eigenvalue.

(13) Taking into account that $\lambda_{0} \neq 0, \alpha=0$.
(14) Vectors $C\left(\lambda_{n}\right)$ defined in (55) take the following values:

$$
C\left(\lambda_{n}\right)=-\frac{32}{\pi^{3}(2 k+1)^{3}}\left(\begin{array}{l}
0 \\
0 \\
1 \\
0
\end{array}\right)
$$

(15) When the minimal theorem [17, page 571] is used,

$$
e^{A u}=\left(\begin{array}{cccc}
e^{2 u} \cosh (u) & e^{2 u} \sinh (u) & 0 & \frac{1}{4} e^{u}\left(3-3 e^{2 u}+2 u\right) \\
e^{2 u} \sinh (u) & e^{2 u} \cosh (u) & 0 & -\frac{1}{4} e^{u}\left(-3+3 e^{2 u}+2 u\right) \\
-e^{2 u} \sinh (u) & -\frac{1}{2} e^{u}\left(-1+e^{u}\right)^{2} & e^{2 u} & \frac{1}{4} e^{u}\left(1+e^{u}\left(-4+3 e^{u}\right)+2 u\right) \\
0 & 0 & 0 & e^{u}
\end{array}\right) .
$$

Next, by considering (74) with $u=-(\pi / 2+n \pi)^{2} t$ and simplifying, we obtain the value of $e^{-(\pi / 2+n \pi)^{2} A t}$.

(16) Values of $C\left(\lambda_{n}\right)$ given in (73) are replaced in (57), and we take into account the value of the matrix $e^{-(\pi / 2+n \pi)^{2} A t}$. After simplification, we finally obtain the solution of problem (10)-(13) given by

$$
\begin{aligned}
& u(x, t) \\
& =\left(\sum_{n \geq 0}-\frac{32 e^{-(1 / 2)(\pi+2 n \pi)^{2} t} \sin ((1 / 2)(\pi+2 n \pi) x)}{\pi^{3}(2 n+1)^{3}}\right) \\
& \quad \times\left(\begin{array}{l}
0 \\
0 \\
1 \\
0
\end{array}\right), \quad(x, t) \in[0,1] \times[0,+\infty) .
\end{aligned}
$$

\section{Conflict of Interests}

The authors declare that there is no conflict of interests regarding the publication of this paper.

\section{Acknowledgment}

This work has been supported by the Generalitat Valenciana GV/2013/035.

\section{References}

[1] R. D. Levine, M. Shapiro, and B. R. Johnson, "Transition probabilities in molecular collisions: computational studies of rotational excitation," The Journal of Chemical Physics, vol. 52, no. 4, pp. 1755-1766, 1970.

[2] J. V. Lill, T. G. Schmalz, and J. C. Light, "Imbedded matrix Green's functions in atomic and molecular scattering theory,"
The Journal of Chemical Physics, vol. 78, no. 7, pp. 4456-4463, 1983.

[3] F. Mrugaía and D. Secrest, "The generalized log-derivative method for inelastic and reactive collisions," The Journal of Chemical Physics, vol. 78, no. 10, pp. 5954-5961, 1983.

[4] M. H. Alexander and D. E. Manolopoulos, "A stable linear reference potential algorithm for solution of the quantum closecoupled equations in molecular scattering theory," The Journal of Chemical Physics, vol. 86, no. 4, pp. 2044-2050, 1987.

[5] V. S. Melezhik, I. V. Puzynin, T. P. Puzynina, and L. N. Somov, "Numerical solution of a system of integro-differential equations arising from the quantum mechanical three-body problem with Coulomb interaction," Journal of Computational Physics, vol. 54, no. 2, pp. 221-236, 1984.

[6] W. T. Reid, Ordinary Differential Equations, Wiley, New York, NY, USA, 1971.

[7] T. Hueckel, M. Borsetto, and A. Peano, Modelling of Coupled Thermo-ElasToplastic Hydraulic Response of Clays Subjected to Nuclear Waste Heat, Wiley, New York, NY, USA, 1987.

[8] J. Crank, The Mathematics of Diffusion, Oxford University Press, Oxford, UK, 1975.

[9] M. D. Mikhailov and M. N. Osizik, Unifield Analysis and Solutions of Heat and Mass Diffusion, Wiley, New York, NY, USA, 1984.

[10] I. Stakgold, Green's Functions and Boundary Value Problem, Wiley, New York, NY, USA, 1979.

[11] E. Navarro, L. Jódar, and M. V. Ferrer, "Constructing eigenfunctions of strongly coupled parabolic boundary value systems," Applied Mathematics Letters, vol. 15, no. 4, pp. 429-434, 2002.

[12] S. L. Campbell and C. D. Meyer Jr., Generalized Inverses of Linear Transformations, Pitman, London, UK, 1979.

[13] V. Soler, E. Defez, M. V. Ferrer, and J. Camacho, "On exact series solution of strongly coupled mixed parabolic problems," Abstract and Applied Analysis, vol. 2013, Article ID 524514, 9 pages, 2013.

[14] C. R. Rao and S. K. Mitra, Generalized Inverse of Matrices and Its Applications, Wiley, New York, NY, USA, 1971.

[15] E. L. Ince, Ordinary Differential Equations, Dover, New York, NY, USA, 1962. 
[16] V. Soler, E. Navarro, and M. V. Ferrer, "Invariant properties of eigenfunctions for multicondition boundary value problems," Applied Mathematics Letters, vol. 19, no. 12, pp. 1308-1312, 2006.

[17] N. Dunford and J. Schwartz, Linear Operators, Part I, Interscience, New York, NY, USA, 1977. 


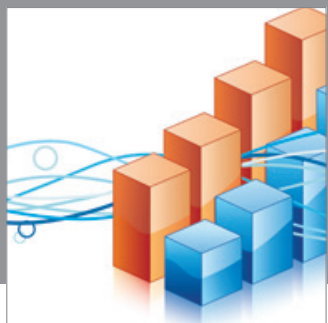

Advances in

Operations Research

mansans

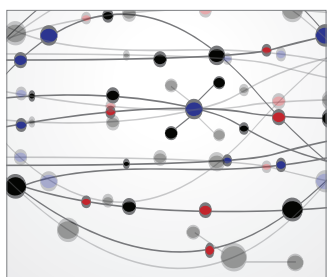

The Scientific World Journal
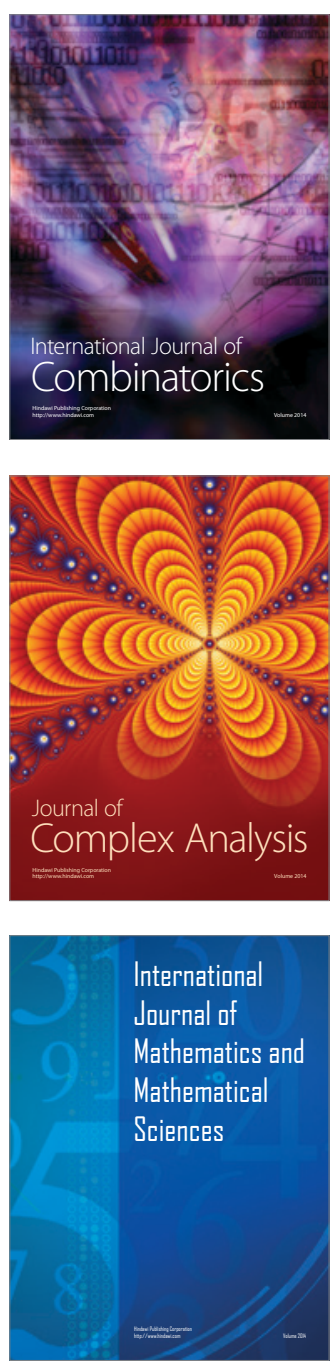
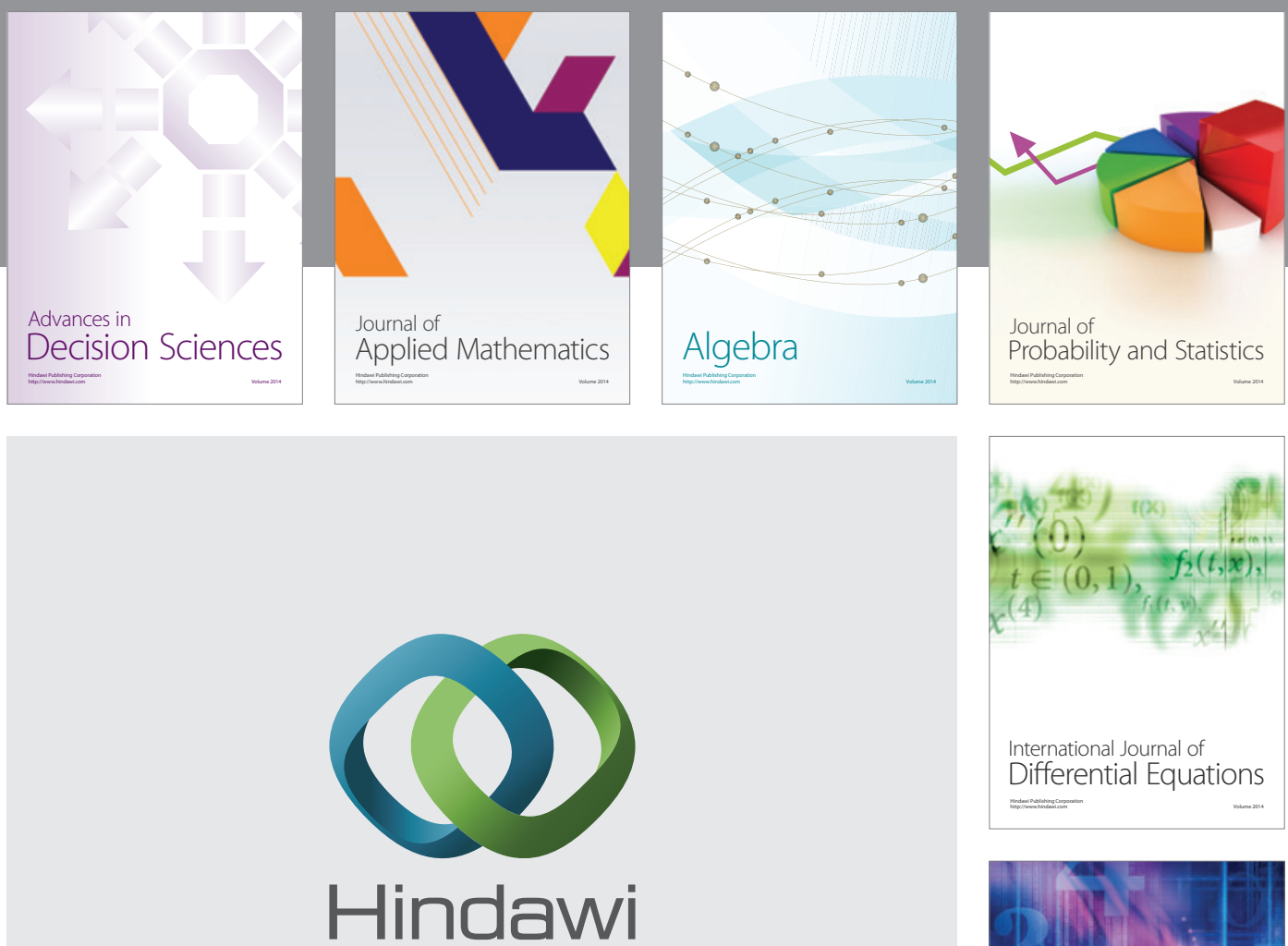

Submit your manuscripts at http://www.hindawi.com
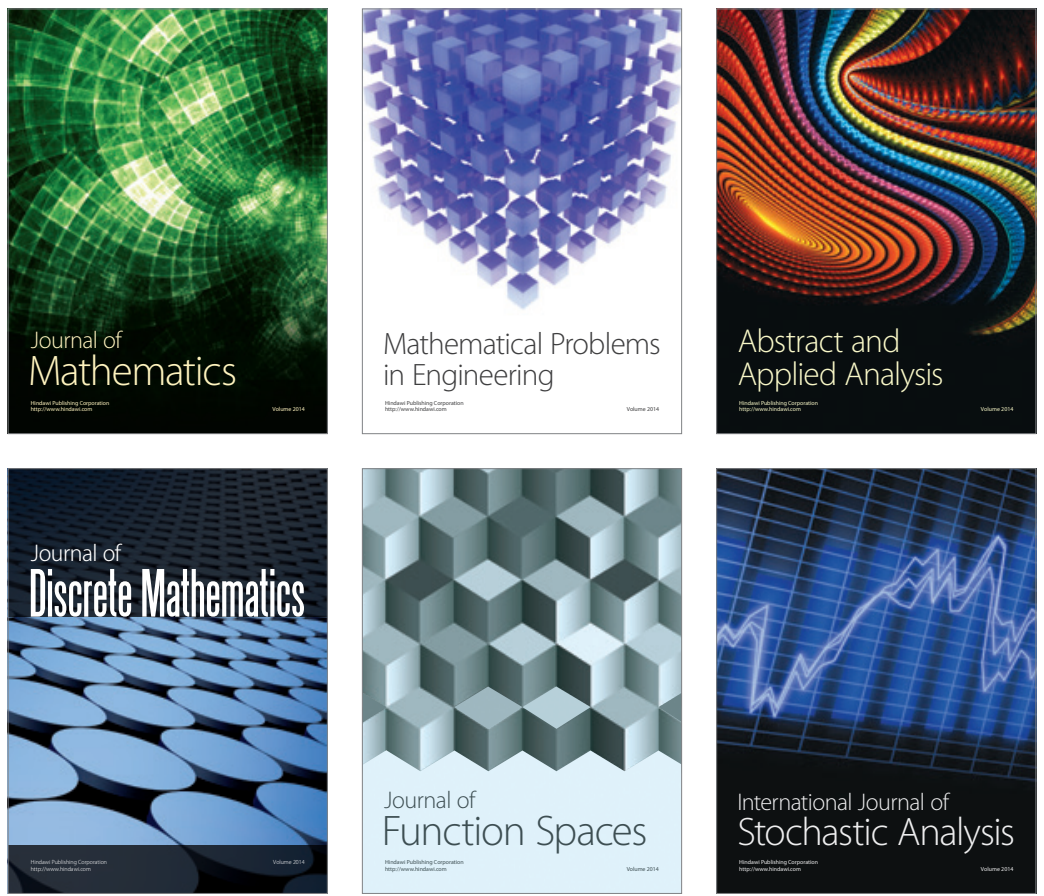

Journal of

Function Spaces

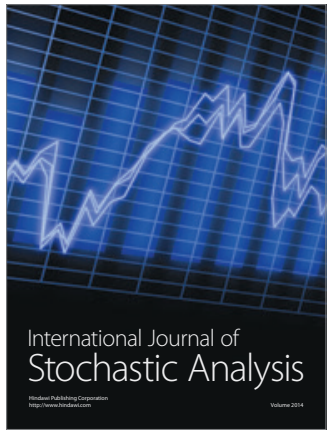

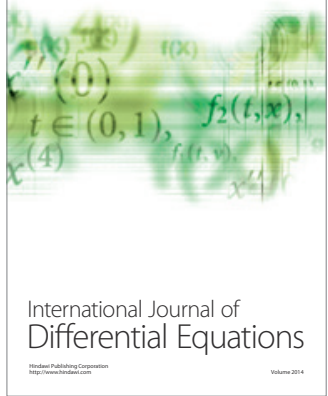
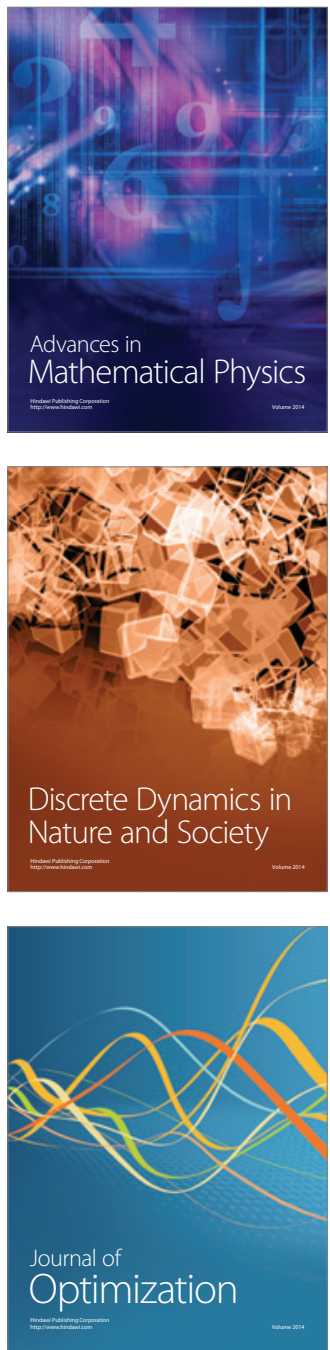